\title{
Gravitational Wave as Symmetry Breaking within a New Model: An Overview
}

\author{
Sandro Antonelli \\ Madonna del Piano 41 A 03017 Morolo (Fr), Italy \\ Email: antonelli41@live.it
}

How to cite this paper: Antonelli, S. (2018) Gravitational Wave as Symmetry Breaking within a New Model: An Overview. Open Access Library Journal, 5: e4549.

https://doi.org/10.4236/oalib.1104549

Received: March 27, 2018

Accepted: April 25, 2018

Published: April 28, 2018

Copyright $\odot 2018$ by authors and Open Access Library Inc.

This work is licensed under the Creative Commons Attribution International License (CC BY 4.0).

http://creativecommons.org/licenses/by/4.0/

\begin{abstract}
I outline a new hypothetical approach issuing a second gravitational equation in the scope of a promising model tackling the gravitational wave problem. This wave equation for graviton is framed in the endeavour to bridge the puzzling missing link to allow for quantum scale physics in a unifying gravity theory, through a new coupling constant $S$ : thus wave is regarded as a symmetry breaking of general covariance of field equations through contraction of Riemann tensor by a constant tensor. That also allows an inertial mass to be assigned to the graviton $\left(O \mathrm{E}-25 \mathrm{eV} / \mathrm{c}^{2}\right)$. This extension of General Relativity stems from self-evident considerations on the differential conditions of compatibility involving the two fundamental tensors on the curvature of the Space-Time continuum. Some considerations about last detected events are broached on the gauging of $S$ constant, bringing forth a value that differs of two orders of magnitude with respect to the fitting of known binary star systems, unless source parameters are revised.
\end{abstract}

\section{Subject Areas}

Theoretical Physics

\section{Keywords}

Gravitational Waves, 2nd Fundamental Tensor, Tensorial Curl, Local and Global Invariance

\section{Introduction}

The latest LIGO and VIRGO's success [1] in marking the first four gravitational wave (GW) signals ever caught [1] [2] [3] [4], while reinforcing testing methods in numerical relativity for the BBH's last stage merging [5] [6], matters in keeping up interest in alternative theories too, though the latest GW170814 event 
makes harder the constraints about the possibility of the mixing presence of non purely 2-spin tensor General Relativity (GR) polarization states [1] [7].

Recently a new equation has been put forward by Tailherer [8] (so-called Vortex model) which expounds in a new very way the genesis and propagation of physical waves by the introduction of another field derived from the metric tensor constituting the $2^{\text {nd }}$ fundamental tensor [9] of Space-Time $K_{\mu \nu}=\partial_{\tau} g_{\mu \nu} / 2 c$, ( $\tau$ proper time) also equal to the deformation speed tensor referred to the 4 -velocity of a testing object $1 / 2\left(\nabla_{h} v_{k}+\nabla_{k} v_{h}\right)^{1}$. What makes the theory compelling is the way it tethers these two fundamental fields by a second gravitational equation (see Equation (1)) through the contrivance of a new universal gravitational constant $(S)$ featuring the intrinsic V4 inertia to spread gravitational energy, (in analogy to heat equation a sort of conductivity or measure of rate of gravitational energy flow for Space-Time continuum) and which allows an inertial mass value to be assigned to the graviton. Aim of this paper is to debate what drags on adding an extra field and a new universal constant to the fundamental symmetry of Einstein's theory for which gravitational equations should remain invariant for general coordinate transformations, and what implies for this prospect to include the attested reality of gravitational waves.

The core ideas of our Model are borrowed from the vorticity gradient formula of continuum mechanics propped up by the device of identifying the vorticity tensor to the contracted Riemann tensor through a constant tensor $\in$ (so non general-globally invariant) and via the constant $S$ (see Equation (1)).

The theory so built turns out not invariant in the sense of general relativity [10] [11], though covariant in the form of equations, just preserving at the most Lorentz invariance for only a particular choice of $\epsilon$ and setting off the special frame in which $S$ is calculated (usually the center of mass system of the system of bodies), but this comes home to our subject by making up a breaking of the invariance of Einstein's gravitational equation from which the GW phenomenon springs out.

Riddling GR inasmuch as insufficient in getting GW's can be gathered this way. As well-known and highlighted f.i. in [12], just from sheer GR an interplay ensues between curvature, determined by the content of any form of energy-momentum through Einstein's equations, and gravitational waves, as usually held. However this would in principle contrast with Weyl's evidenced GR feature of plasticity of the continuum Space-Time and non-consistence with it of physical ripples of metric as shown in [13] [14]: actually, it suffices choosing four suitable components of the metric tensor at our disposal in passing to a new gauged manifold (for only interacting gravitational forces just a co-moving reference frame) for which the motion of a dust particle can be geodetically blotted out [15]. Alternatively, assuming Fermi's coordinates, the metric tensor is

\footnotetext{
${ }^{1}$ We take occasion to remark a slip in [9] where we talked about the 2 nd rank $K_{\mu v}$ symmetric tensor as a 2 spin-2 field. Strictly speaking, being not traceless, it cannot be so, but rather it has a spurious decomposition in angular momenta as $0 \oplus 2$ in gaussian gauge $\left(g_{0 i}=0, g_{0 i}=1\right)$, so in all $\left(2 j_{1}+1\right)+\left(2 j_{2}+1\right)=6$ modes being reduced to 2 by 4 further accessory boundary conditions.
} 
self-parallel transported according to Fermi-Walker on the world line, geodesic or not, leaving out the chance of gravitational radiation, $\mathrm{d}^{F W} g_{\mu v} / \mathrm{d} t=0$ implying $g_{\mu v}=$ constant.

\section{Further Shortcomings of GR}

From a mathematical point of view, it can be shown that the characteristic hypersurfaces of Einstein's equations, implying a discontinuity being propagated so-called a wave-, coincide with the light hypercone [16] [17], i.e. a developable hypersurface whose Riemann tensor vanishes identically, that is to say a constant metric can be assigned on a light ray as well.

As to back-reaction ${ }^{2}$ of gravitational radiation [18] [19] in the full non-linear theory in medium-strong regime $\left(\mathrm{Gm} / \mathrm{rc}^{2} \geq 1 / 5\right)$ based on Post-Newtonian expansion (near zone) and backed up by the sophisticated post-Minkowskian one (far zone) by Blanchet-Damour, would want for an existence theorem ${ }^{3}$ for which these configurations converge to the exact solutions of Einstein's equations, even worse unknown for the two body problem. Moreover these expansion methods could be questioned by the simple argument of energy conservation of motion for any closed system, whose mechanics is unequivocally characterized by the travelled geodesics of material bodies, in addition to the continuity equation (we recall the apsidal precession derived among others in Schwarzschild metric just from the energy's integral of motion). Thus an inspiralling trajectory would mismatch a geodesic not as much differently a lexodrome (as known not geodetic) would onto a bi-dimensional sphere. Furthermore, still admitting a wave propagation phenomenon, it can be shown that for a reference frame riding the wave front, the metric tensor is practically time-independent and consequently, by Serini's theorem, Serini's theorem for which the constant metric is the only regular Einstein's solution, the Riemann curvature tensor vanishes, hence carrying no metric undulation.

\section{Vortex Model of GW}

Accordingly, the only presence of a curvature $\left(R_{\mu v \rho \sigma} \neq 0\right)$ would not necessarily imply a propagating field, what as to Vortex theory there would make a gradient of curvature instead or, terming it with GR's parlance by the equivalence principle, a gradient of acceleration. For plainness, let us lay down Tailherer's equation got identifying, up to a constant, the 4-vorticity $\omega_{\mu v}$ with the skew tensor $C_{\mu \nu}=R_{\mu \nu \alpha \beta} \in^{\alpha \beta}$ (dubbed skew $C$-tensor), i.e. the ansatz $\omega_{\mu \nu}=C_{\mu \nu} / S$ with $\epsilon^{\alpha \beta}=1$ for $\alpha, \beta$ consecutive indexes and $\epsilon^{\alpha \beta}=-\epsilon^{\beta \alpha} \quad$ (det $\in=1$ )

$$
\nabla_{\sigma} C_{\mu \nu}=S\left(\nabla_{\mu} K_{v \sigma}-\nabla_{v} K_{\mu \sigma}\right)(\mu, v, \sigma=1,2,3,4)
$$

We assumed for the $\epsilon^{\alpha \beta}$ matrix to transform as a contravariant tensor even ${ }^{2}$ Contributions starting from $2.5 \mathrm{PN}$ terms i.e. $\mathrm{O}\left(1 / c^{5}\right)$.

${ }^{3}$ Even in math we know functions whose expansion does not tend continuously to a point limit as f.i. $\exp (-a / x)=1-(a / x)+1 / 2(a / x)^{2}+\cdots$ that for $a>0$ gets worse with the order for $x$ tending to 0 , while the limit for the starting function equals 0 being quite regular in its neighborhood. 
though it is not invariant for a general coordinate transformation, so the $x^{\mu}\left(x^{\prime \mu}\right)$ frame in which we calculate $S$, i.e. the center of mass system, takes on a special meaning: it is a privileged frame whose track cannot be deleted by a transformation that so keeps trace of it. We mean to say that $\frac{\partial x^{\prime \rho}}{\partial x^{\alpha}} \frac{\partial x^{\prime \sigma}}{\partial x^{\beta}} \epsilon^{\alpha \beta} \neq \epsilon^{\alpha \beta}$ but performing another coordinate transformation we see that $\frac{\partial x^{\prime \prime}}{\partial x^{\prime}} \frac{\partial x^{\prime \delta}}{\partial x^{\alpha}} \frac{\partial x^{\prime \prime \sigma}}{\partial x^{\prime \gamma}} \frac{\partial x^{\prime \gamma}}{\partial x^{\beta}} \epsilon^{\alpha \beta}=\frac{\partial x^{\prime \prime}}{\partial x^{\alpha}} \frac{\partial x^{\prime \prime \sigma}}{\partial x^{\beta}} \epsilon^{\alpha \beta}$ and last expression has the same form than the previous one (covariance) except that the $x^{\mu}$ appertain to a peculiar frame, i.e. we got local covariance. Namely, Equation (1) is not a general invariant law as Einstein's equation, so that we might say that GW's make up a symmetry breaking of the general relativistic covariance, as it should be since we have showed how Einstein's theory, in his own formulation, would not actually entail physically and energetically consistent wavy solutions. Indeed only light waves obey globally invariant formulation. Given the arbitrariness in choosing $\epsilon^{\alpha \beta}$, had we singled out for it the tensor $\epsilon^{\prime \alpha \beta}=\left(\begin{array}{cccc}0 & 1 & 0 & 0 \\ -1 & 0 & 0 & 0 \\ 0 & 0 & 0 & 1 \\ 0 & 0 & -1 & 0\end{array}\right),\left(\operatorname{det} \epsilon^{\prime}=1\right)$, we would also have kept invariance with respect to Lorentz transformations, which might provide in case a suitable starting ad-hoc inertial frame to assess the constant $S$. The $S$ gauged (cf. [9] \$3.2) by this option associated with Equation (1) would have been of the same order of magnitude than our evaluated $S=(2.5 \pm$ 1.2) $\mathrm{E}-19 \mathrm{~m}^{-1}$, got averaging the fitting of the orbital energy loss decay of four known binary systems ${ }^{4}$ [20] (see Figure 1 for the B1913 +16 period fitted decay). This would brand the passage from classical relativity into quantum gravity-likewise on turn Planck constant did with respect to classical mechanics-through the gauging of the length of interaction involved, represented by the Compton length $S^{-1}=h / m_{G} c$ referred to the inertial mass of graviton $m_{G}=(3 \pm 1) \mathrm{E}-25 \mathrm{eV} / c^{2}$, so tested to be within the bound found for GW150914 [4] $\left(<7.7 \mathrm{E}-23 \mathrm{eV} / \mathrm{c}^{2}\right)$.

By doubling the tensorial curl (incomplete) of Equation (1) the radiative behaviour becomes evident in the RHS getting a $\square-\operatorname{grad}($ div) operator ([8], eq.4.18) on $K_{\mu v}$ (to note a d'Alambertian is a 4 dimensional laplacian). In spite of the hypothetical massive gravity equation for a 2-spin tensor derived from the Proca-Fierz action [21], this one does not subrogate Einstein's one (anyway linearized), but would endow it with the right radiative-dissipative feature. Now, it is known how to relate the metric tensor to the matter via Einstein's equation through the Ricci tensor, but there is not a way to elicit from it the full Riemann tensor $R_{\mu \nu \rho \sigma}$ to yield the $C_{\mu \nu}$ tensor. The best that can be done is to estimate it as ${ }^{(1)} R_{\mu v a \beta}=g_{\mu \beta} R_{v a} / 4$ just because of $R_{v a}=R_{\rho v a \sigma} g^{\rho \sigma}$ so being not a sufficient condition to get it (actually it corresponds to approximate a tensor with its trace times a

${ }^{4}$ The best fit for the relativistic system J0737-3039 provides an agreement in a period change with extrapolated experimental data that can barely be discriminated to within the approximation precision, despite the coarse starting assumptions for the curvature tensor. 

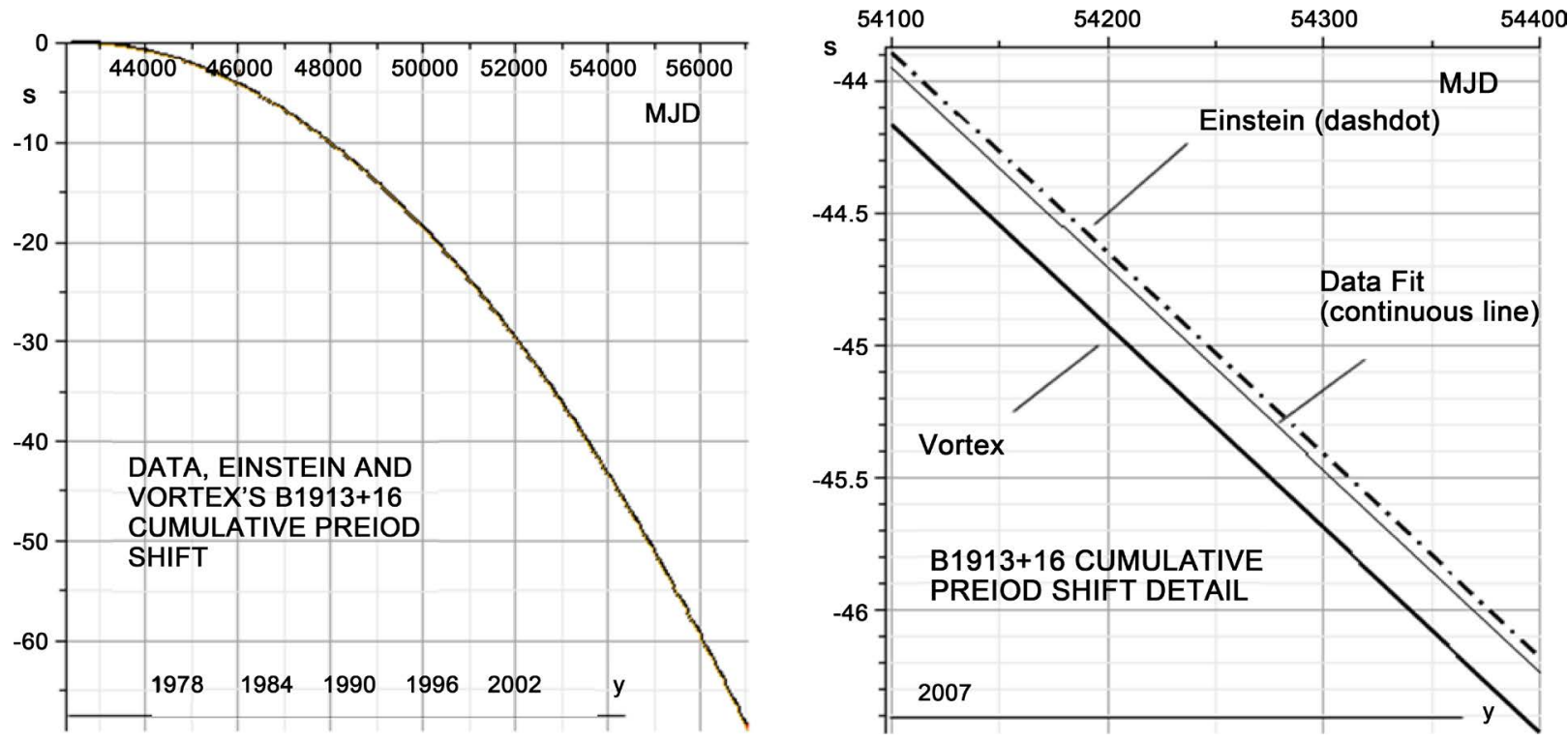

Figure 1. B1913 + 16 period change in the damping of system paralleling GR, Vortex prediction and fitted data vs Modified Julian Day Time from 1975 to 2013. On sight the three functions cannot be distinguished. By magnifying we can observe how the GR approximates closer up to $0.2 \%$ to the data in comparison with Vortex Theory prediction which indeed is seen in the detail inset to anticipate of about $0.5 \%$ (After [20]).

term proportional to the metric tensor). Rather, it is a stopgap to insert the energy-momentum into the $C_{\mu v}$ tensor by means of Einstein's equation viz. $C_{\mu \nu}=-\frac{8 \pi G}{4 c^{4}} g_{\alpha \nu}\left(T_{\beta \mu}-\frac{1}{2} g_{\beta \mu} T\right) \epsilon^{\alpha \beta}$ as starting position of a recursive method to build $R_{\rho v a \sigma}$ solving Equation (1). Computing the latter has been proved decisive in the calculations [20]. Therefore, we have not only the gravitational field $g_{\alpha \beta}$ linked to the energy-momentum as from GR but also $K_{\mu v}$ by means of its gradient $\left(C_{\mu \nu} \propto R_{\mu \nu \rho \sigma} \propto T_{\mu \nu}\right.$ ), thus bearing on energy wave propagation, namely radiated energy related to the action density $-1 / 4 \Phi_{\alpha \sigma \rho} \Phi^{\alpha \sigma \rho}$ whose field $\Phi_{v \sigma \mu}=K_{v \sigma / \mu}-K_{\mu \sigma / v}$. Indeed, the problem well put is to get $K_{\mu \nu}$ from Equation (1) and then $g_{\alpha \beta}$ from integration with respect to the proper time.

We dwell on the form of the integral solution at large distance $r$ ([8], equ.4.20a):

$$
K_{\mu \nu} \approx-\frac{1}{\pi^{2} S} \int \frac{S_{\mu \nu}\left(x^{\prime}\right)}{\left[\left(x_{0}^{\prime}-x_{0}\right)^{2}-\left|\vec{x}-\vec{x}^{\prime}\right|^{2}\right]} \mathrm{d}^{4} x^{\prime}=-\frac{1}{\pi^{2} S r^{2}} \int-S_{\mu \nu}\left(x^{\prime}\right) \mathrm{d}^{4} x^{\prime}
$$

with $S_{\mu v}$ source forcing term depending on energy-momentum $T_{\mu v}=\Sigma_{i} \int \mathrm{d} t_{s} \delta^{4}\left(x^{\rho}-x_{1}^{\rho}\right) m_{i} u^{\mu} u^{v}$.

The integrand is meant as function of the Nth iterated ${ }^{N} K_{\mu \nu}$ reckoned starting from ${ }^{(0)} K_{\mu \nu}=0$ i.e. ${ }^{(0)} g_{\mu \nu}=2 c \int K_{\mu \nu} \mathrm{d} \tau=\eta_{\mu \nu}$, Minkowski metric. As for the case of quoted four binaries we stopped to the $N=1$ stage.

On first inspection, this solution might appear improper because $1 /\left[c^{2}\left(t_{0 s}^{\prime}-t_{o b}\right)^{2}-r^{2}\right]$ as depending on integration variables could not be taken out of the integral naively as $1 / r^{2}$ by evaluating it at the same times in gaussian gauge. The correct solution would be then to consider the previous solution as 
Green function $G\left(x_{0 s}-y_{o b}\right)=1 /\left[c^{2}\left(t_{0 s}-t_{o b}\right)^{2}-r^{2}-i \varepsilon\right]$ between two points $x_{0 s}$ (source) and $y_{o b}$ (observer) of the Space-Time at different instants and integrate with respect to source time just along a closed contour in the complex plane as usual for propagators, with respect to the pole $t_{0 s}-t_{o b}=-r / c$ what gives the retarded potential $1 / r$.

Nevertheless differently from electromagnetism where a space 4-current density distribution enter the integrand letting the time variable to be integrated in the complex plane, here rather a stark 4-dimensional problem is concerned, namely any component the Riemann tensor gradient different from zero could, at least ideally, represent a source term on LHS of Equation (1); thus technically we cope with a 4-dimensional Dirac source term in the Green function rather than a 3 -dimensional one, what involves an $1 / s^{2}$ solution of a $2^{\text {nd }}$ order PDE taken in account of the homogeneity property of Dirac delta $\delta(\underline{s} \underline{x})=\delta(\underline{x}) / s^{4}$.

Indeed as also checked a delayed potential $1 / r$ is not at all advisable out of the computational bulking not only linked to the rather mild iterative convergence.

Thereby in order to avoid the denominator singularity without performing the integral in $\mathrm{d}_{0 \mathrm{~s}}^{\prime}$ in the complex variable there can be exploited the translational invariance of a problem about quasi-periodic system like in the case of binaries, by evaluating the integrand at the time $t_{0 s}^{*}=t_{0 s}+r / c=t_{o b}$ at the cost of an additive phase factor in $S_{\mu v}\left(x^{\prime}\right)$ immaterial when averaged over the period in getting the radiated energy loss, but such that the difference $t_{0 s}^{*}-t_{o b}$ elides at the denominator leaving just $1 / r^{2}$, which definitely betters the iteration convergence. Evidently, the case of a burst does not lend itself to this ploy.

Thus working out the solution in this way gives more significant results just from the first approximation. Worth noting is that the radiated energy associated with the $K_{\mu \nu}$ wave running as $1 / r^{2}$ still gets conserved provided that the related inferred metric be considered in the flux of the energy-momentum and in rising/lowering indexes therein. This comes from the relation $\nabla_{v} t_{\mu}^{v}=0$ i.e. $\frac{1}{\sqrt{-g}} \frac{\partial\left(t_{\mu}^{\nu} \sqrt{-g}\right)}{\partial x^{\nu}}-\frac{1}{2} \frac{\partial g_{v \rho}}{\partial x^{\mu}} t^{\nu \rho}=0$ and on disregarding the last term on $\operatorname{LHSO}\left(1 / r^{3}\right)$, the quantity $\int t_{\mu}^{0} \sqrt{-g} \mathrm{~d}^{3} V$ gets conserved.

\section{Some Fingerprints of the Model}

What is peculiar in this theory is that a cosmological constant does not contribute the radiation field nor a constant energy momentum tensor (f.i. the electromagnetic cosmic background energy density, excluding anisotropy), so retrieving the static condition satisfied by the $2^{\text {nd }}$ fundamental tensor with respect to crossed derivation, that is to say the

Mainardi-Codazzi conditions [22] [23] (let us recall that for a globally isotropic manifold the expression for the Riemann tensor holds:

$R_{\mu \nu \rho \sigma}=\mathfrak{K}\left(g_{\mu \rho} g_{v \sigma}-g_{\mu \sigma} g_{v \rho}\right)$ whose gradient vanishes because of the Schur's theorem [24]).

That the notion of curvature inherent in the Riemann tensor is not sufficient 
to characterize the evolution of continuum Space-Time can also be seen from the fact that for an ordinary surface the local curvature of a generic section depends not only on Gaussian curvature $\mathfrak{K}=c_{1} \cdot c_{2}$ but also on pseudo-invariant expressed by the trace $H=K_{\alpha}^{\alpha}=c_{1}+c_{2}$ via Euler curvature formula [25]: $c=c_{1} \cos ^{2} \theta+c_{2} \sin ^{2} \theta=\frac{H-\sqrt{H^{2}-4 \mathfrak{K}}}{2} \cos ^{2} \theta+\frac{H-\sqrt{H^{2}-4 \mathfrak{K}}}{2} \sin ^{2} \theta$, thus still two parameters, ( $c_{1 / 2}$ principal curvatures and $\theta$ the section angle with respect to the first one).

From a mere kinematic point of view we may express as well the variation of the 4-velocity of a test moving object in the continuum Space-Time [26] as act of motion with vierbein representation $\boldsymbol{e}^{v}$ i.e. $.^{5} \delta \boldsymbol{v}=\left(K_{\mu v}+\omega_{\mu v}\right) \boldsymbol{e}^{v} \delta x^{\mu}$ or by means of Tailherer's ansatz $\left(K_{\mu v}+C_{\mu v} / S\right) \boldsymbol{e}^{v} \delta x^{\mu}$, thus evidencing the need of considering both the tensors for a full description of motion, $C_{\mu \nu}$ including all kind of interactions underlying the Riemannian manifold. However, the last expression says more on the structure of continuum Space-Time, because even if the gradient of $C_{\mu \nu}$ vanished and so there were no gravitational radiation according to Equation (1), in the first order neighbourhood of any point there would yet appear the quantity $S$ as a characterizing mark of curved Space-Time.

Because we are dealing with a $0 \oplus 2 K_{\mu v}$ field (note 1), there are extra-modes rather than 2 that can be taken away by suitable null boundary radiation conditions at infinity as instanced in ([9], \$2.3) in Minkowsky referred background asymptotic metric.

More specifically the hallmark of this theory versus GR occurs in the polarization mode $\oplus\left([20]\right.$, Appendix) whose strain $h_{\oplus \text { Vortex }}=\left|\begin{array}{ll}1 & 0 \\ 0 & 1\end{array}\right|$, differently from GR $\oplus$ mode $\left|\begin{array}{cc}1 & 0 \\ 0 & -1\end{array}\right|$, would cause equal phase displacement of laser path in LIGO-like detectors, so space symmetric events like supernova explosions would need other apparatus than Michelson interferometry that would turn out blind to them at normal GW impact. Then an alternative new type has been sketched in ([9], \$4). Moreover it predicts the lack of the uncoupling of the $\otimes$ and $\oplus$ polarization modes $([9] \S 2,3)$ in correspondence of edge-on line of sight of binary systems (this latter $\oplus$ polarized according to linearized GR [27]). This is for checks to come. Apparently, according to Vortex theory, recent detection of GW events would be ascribed chiefly to the $\otimes$ mode.

Further, pointing out the analogy between Equation (1) and Faraday-Neumann equation in electromagnetism, we could correspond the $E$ s and $B$ s vector role to the $K_{\mu \nu}$ and $g_{\mu \nu}$ tensors (the latter through the Riemann $C$-tensor) by saying that a variation of energy-momentum linked to $C_{\mu v}$ (LHS of Equation (1)) would cause ${ }^{5}$ This is promptly seen by taking the gradient of 4 -velocity $\partial_{h} v=q_{h k} e^{k}$ whose matrix $q_{h k}$ can always be split up in a symmetrical part and a skew-symmetric one identified by the deformation speed tensor and 4-vorticity $q_{h k}=\partial_{h} v \cdot \boldsymbol{e}_{k}=K_{h k}+\omega_{h k}$.

${ }^{6}$ Let recall the Green identity $\oint K_{\mu \sigma} \mathrm{d} x^{\mu}=\frac{1}{2} \iint \mathrm{d} f^{\mu v}\left(\partial_{\mu} K_{v \sigma}-\partial_{v} K_{\mu \sigma}\right)$, with $\mathrm{d} f^{\mu v}$ 4-facet surface element. 
a propagation of the field $K_{\mu \nu}$ over the Space-Time (RHS), and vice versa, by applying the Green-Stokes theorem ${ }^{6}$ to Equation (1) for a sufficiently large 2-surface $\Sigma$ enclosing the mass system (for details see Equation (4) in [9])

$$
\oint K_{\mu \sigma} \mathrm{d} x^{\mu} \approx \nabla_{\sigma} \Phi_{\Sigma}\left(C_{\mu \nu}\right) / S(\sigma=1,2,3,4)
$$

it holds true that a cyclic discontinuity in the circulation of $K_{\mu \nu}$ would cause a variation of the flux of energy-momentum through the Riemann $C$-tensor.

\section{A Glance at Recent Discoveries}

Finally, applying the Vortex Model in the non relativistic regime to the GW150914 event for the circularized black-holes'orbit and on retaining the estimated source parameters, outputs a fitted value $S=1.7 \mathrm{E}-17 \mathrm{~m}^{-1}$ (corresponding to graviton mass $m_{G}=h S / c=2 \mathrm{E}-23 \mathrm{eV} / c^{2}$ ) at the GR's luminosity peak of $3.6 \mathrm{E} 49 \mathrm{~J} / \mathrm{s}$ [2], hence two orders of magnitude larger than that of binary pulsars' analysis, unless involved masses be lowered and/or source distance be increased. (Of course radiated power should not depend on distance from source but in the approximated theory it does). Keeping to that value for $S$ would imply a given out luminosity of $O(1 \mathrm{E} 60) \mathrm{J} / \mathrm{s}$ apparently unacceptable. A similar result is obtained for GW151226 outputting a fitted $S=3 \mathrm{E}-17 \mathrm{~m}^{-1}$ whilst $1.7 \mathrm{E}-17 \mathrm{~m}^{-1}$ and $1.9 \mathrm{E}-17 \mathrm{~m}^{-1}$ concerning GW170104 and GW170814 events, which confirms a systematic drawing away of GR from Vortex Model's expectations. Indeed, the binary pulsar's estimate for $S$ is to be accepted as the more reliable one, as upheld on collation of observational astronomical data and non-radiative general relativistic effects (up to $2 \mathrm{PN}$ ) such as periastron advance and Shapiro or Einstein's delay [28]. Anyway for BBH's events, a thorough general relativistic treatment encompassing calculation of $K_{\mu \nu}$ at the further recursive stage requires bigger computational efforts in terms of available RAM ( $>60 \mathrm{~Gb})$, which nevertheless should be carried out for a comprehensive description with respect to curved background metric provided by the reckoning step.

\section{Conclusions}

We have briefly reviewed a modified gravity theory based on the introduction of the new fundamental $2^{\text {nd }}$-rank symmetric field $K_{\mu v}=\partial_{t} g_{\mu v} / 2 c$ and a consequently $3^{\text {rd }}$-rank skew field $\Phi_{v \sigma \mu}=K_{v \sigma / \mu}-K_{\mu \sigma / v}$ in the action (mimicking strength tensor in electromagnetism), that among other things we deem as requisite on grounds of differential geometry (Sect. 4) in completing Einstein's description and in particular the gravitational wave phenomenon. Indeed, gravitational wave is seen as a break of the general invariance as implied by Einstein's theory whose stint, as discussed in the Sect. 1 - 2, would consist in describing only a conservative system that does not lose gravitational energy: actually, as remarked in [13] [14] [15] it is impossible to admit a physical wave and general invariance. The experimented radiative phenomenon therefore would ultimately mean in dynamical gravity to overcome this stark einstenian principle towards local frame-dependent invariance entailed by an ad-hoc 
privileged coordinate system relevant to an appropriate "seed" metric in which we calculated the new universal constant $S$ featuring the specific inertial property of Space-Time to spread gravitational radiation. Mathematically this translates into the property of local invariance owned by the Riemann tensor saturated with a constant skew-symmetric tensor $R_{\mu v \alpha \beta} \in^{\alpha \beta}$. This leads to the Vortex equations of the model (Equation (1)) constituting a differential link between the field $K_{\mu \nu}$ and the aforementioned Riemann curvature contracted tensor (Sect. 3).

Direct resolution of vortex equations through Kelvin-like integral Equation (2) [cf. also [20], Equation (1)] relied on successive approximations for catalogued binary systems, thus proving very clumsy. Nevertheless it puts forth an estimate for $S=3 \mathrm{E}-19 \mathrm{~m}^{-1}$ differing by two orders from recent discovery events (Sect. 5), but still within the related experimental inferred graviton's mass bound $O \mathrm{E}-23$ $\mathrm{eV} / \mathrm{c}^{2}$. Therefore one could think that exploiting the integral version of Vortex equations-Equation (3) would lighten the calculus burden for $K_{\mu \nu}$ bettering the valuation for $S$, but at this time it is not at all evident how to manage it profitably.

And yet the better insights of the theory have to be expected in applying the model to the cosmological problem solving the equations with respect to the FLRW initial metric, since all the cosmological constant energy density conundrums, as noted before in Sect. 4, get superfluous in determining the evolution of the universe scale factor, inflationary ones included.

\section{References}

[1] LIGO-VIRGO coll. (2017) GW170814: A Three-Detector Observation of Gravitational Waves from a BBH Coalescence. Preprint arXiv:gr-qc/1709.09660.

[2] Abbott, B.P., et al. (2016) Observation of Gravitational Waves from BBH Merger. Physical Review Letters, 116, 061102.

[3] LIGO coll. (2016) GW151226: Observation of Gravitational Waves from a 22 Solar-Mass BBH Coalescence. Physical Review Letters, 116, 241103.

[4] Abbott, B.P., et al. (2017) GW170104: Observation of a 50-Solar-Mass BBH Coalescence. Physical Review Letters, 118, 221101.

[5] Lovelace, G., et al. (2016) Modeling the Source of GW150914 with Targeted Numerical-Relativity Simulations. Classical and Quantum Gravity, 33, 244002. Preprint arXiv:gr-qc/1607.05377.

[6] LIGO coll. (2016) Test of GR with GW 150914. Preprintar Xiv:gr-qc/1602.03841.

[7] DeLaurentis, M., et al. (2016) Constraining Alternative Theories of Gravity Using GW. Preprint arXiv:gr-qc/1611.05766.

[8] Tailherer, M. (2007) A Critical Reading on the Theory of Gravitational Wave Propagation. Journal of Physical \& Natural Sciences, 1, 1.

[9] Antonelli, S. (2014) Outstanding Outcomes from a Recent Theory of Gravity. International Journal of Physics, 2, 267-276. https://doi.org/10.12691/ijp-2-6-10

[10] Anderson, J.L. (1967) Principles of Relativity Physics. Academic Press, New York.

[11] Norton, J.D. (1993) General Covariance and the Foundations of General Relativity: Eight Decades of Dispute. Reports on Progress in Physics, 56, 791-458. https://www.pitt.edu/ jdnorton/papers/decades.pdf 
[12] Cooperstock, F.I. (2015) The Essence of Gravitational Waves and Energy. International Journal of Modern Physics D, 24, 1543005. https://doi.org/10.1142/S0218271815430051

[13] Weyl, H. (1988) Raum-Zeit-Materie. Springer, Berlin, p. 268, quoted in Loinger. A. (2007) GW’s towards Fundamental Principles of GR.

[14] Loinger, A. and Marsico T. (2016) Remarks on Numerical Relativity, Geodesic Motions, Binary Neutron Star Evolution. Preprint arXiv:gen-ph/1211.6152.

[15] Loinger, A. and Marsico, T. (2010) All Relativistic Motions Can Be Relativistically Described. arXiv:gen-ph/1006.3844.

[16] Ferrarese, G. (2001) Lezioni di Relatività Generale. Pitagora Eds., Bologna, Ch. 10.4, p. 333.

[17] Levi-Civita, T. (1930) Caratteristiche e bicaratteristiche delle equazioni gravitazionali di Einstein. Rendiconti Accademia dei Lincei, 6, 3-11, 113-121.

[18] Maggiore, M. (2008) Gravitational Waves. Vol. 1, OUP, Oxford, §5.3.5.

[19] Blanchet, L. (2010) Post-Newtonian Theory and the Two-Body Problem. Preprint arXiv:gr-qc/0907.3596 appeared in Mass and Motion in General Relativity, Proceedings of the C.N.R.S. School in Orleans, France edited by Springer, Berlin.

[20] Antonelli, S. (2016) Appraisal of a New Gravitational Constant. The International Journal of Physics, 3, 139-149.

[21] Padmanabham, T. (2008) From Gravitons to Gravity: Myths and Reality. International Journal of Modern Physics D, 17, 367.

[22] Weisstein, E.W. (1998) Peterson-Mainardi-Codazzi Equations. From MathWorld-A Wolfram Web Resource. http://mathworld.wolfram.com/Peterson-Mainardi-CodazziEquations.html

[23] Do Carmo, M.P. (1976) Differential Geometry of Curves and Surfaces. Prentice-Hall, New York, p. 235.

[24] Ferrarese, G. (2001) Lezioni di Relatività Generale. Pitagora Eds., Bologna, Ch. 2.13, p. 57.

[25] Do Carmo, M.P. (1976) Differential Geometry of Curves and Surfaces. Prentice-Hall, New York, sec. 3.3, p. 145.

[26] Ferrarese, G. and Stazi, L. (1989) Lezioni di Meccanica Razionale. Vol 2, Pitagora Eds., Bologna, ch. VIII, \$1.8, p. 593.

[27] Maggiore, M. (2008) Gravitational Waves. Vol. 1, OUP, Oxford, sec. 3.6, Prb.3.2, p. 159.

[28] Maggiore, M. (2008) Gravitational Waves. Vol. 1, OUP, Oxford, sec. 6.2.3. 